\title{
Appearance based Background Subtraction for PTZ Cameras
}

\author{
Hasan Sajid, Sen-ching S. Cheung, and Nathan Jacobs ${ }^{a}$ \\ ${ }^{a}$ Center for Visualization and Virtual Environments, University of Kentucky
}

\begin{abstract}
Traditional background subtraction algorithms assume the camera is static and are based on simple per-pixel models of scene appearance. This leads to false detections when the camera moves. While this can sometimes be addressed by online image registration, this approach is prone to dramatic failures and long-term drift. We present a novel background subtraction algorithm designed for pan-tilt-zoom cameras that overcomes this challenge without the need for explicit image registration. The proposed algorithm automatically trains a discriminative background model, which is global in the sense that it is the same regardless of image location. Our approach first extracts multiple features from across the image and uses principal component analysis for dimensionality reduction. The extracted features are then grouped to form a Bag of Features. A global background model is then learned from the bagged features using Support Vector Machine. The proposed approach is fast and accurate. Having a single global model makes it computationally inexpensive in comparison to traditional pixel-wise models. It outperforms several state-ofthe-art algorithms on the CDnet 2014 pan-tilt-zoom and baseline categories and Hopkins155 dataset. In particular, it achieves an F-Measure of $75.41 \%$ on the CDnet dataset PTZ category, significantly better than the previously reported best score of $62.07 \%$. These results show that by removing the coupling between detection model and spatial location, we significantly increase the robustness to camera motion.
\end{abstract}

Keywords: background subtraction, foreground detection, image segmentation.

\section{Introduction}

Background subtraction (BS) is the algorithmic process that segments out the region of interest often known as Foreground (FG) from the Background (BG). It is one of the pre-processing steps in many vision applications such as object tracking, object recognition, surveillance, behavior recognition, etc. It is a wellresearched area in computer vision with significant amount of literature [1]. The focus of most research in background subtraction has been on stationary cameras, whereas PTZ cameras have become increasingly popular because of the wide area coverage and additional perspective it offers. Existing algorithms fail in the case of moving camera as neither FG nor BG pixels are stationary.

There are three general approaches for BS: 1) model-based, 2) motion-based and 3) hybrid motion and model based algorithms. Model-based approaches construct a model of the background and then compare the pixels of an input image with the model to classify them as FG or BG. The main assumption of these algorithms is that the camera remains static with moving FG while building the BG model. Hence, BS algorithms are unsuitable to handle moving cameras.

The motion-based algorithms rely on the assumption that for any given number of images, the foreground moves differently from the background [2,3]. These algorithms exploit the difference in motion patterns to segregate FG from the BG. These algorithms assume rigid motion in all BG regions. This is however not true since dynamic background can comprise of nonrigid motions such as waving trees. Another challenge is when the foreground object itself is at rest and follows the same camera motion as the rest of the static background. Other motionbased algorithm estimates background by finding regions that do not change in the sequence [4]. While it alleviates the static foreground problem, such an approach would fail in the case of dynamic background.

The last category i.e. model and motion based methods such as [5] and [6] combines both appearence and motion information in an online framework. Motion information in the intial frames is used to initialize FG and BG appearence models, which are then continuously maintained and updated overtime. Classification is done using the appearence model. In general these methods require special intialization procedures and are computationaly expensive.

In this paper, we propose a novel BS algorithm named BoFsSVM that can robustly identify FG objects from PTZ camera sequences. Our algorithm learns the background entirely based on the appearance features and their attributes extracted by a sliding window over each pixel to encode the BG into a Bagof-Features (BoFs). The sliding window captures spatial dependencies at the local level. The extracted features then undergo Principal Component Analysis (PCA). The selected features are then concatenated into feature vectors to train a single SVM for classification. Our algorithm has a number of unique traits to handle camera movement due to PTZ. The extraction of multiple features from image patches instead of individual pixels is the first key trait that makes our algorithm robust to a moving camera. The second key trait is the absence of any global spatial constraint on the features. This is advantageous 
for moving camera scenarios where BG is continuously changing and spatial constraints do not hold. This is in constrast to existing methods which impose spatial constraint and maintain individual models for each pixel. The selection of the most informative, scene-specific feature set is the third key trait of our algorithm. To avoid the ambiguity of dynamic background and static foreground, our work focuses exclusively on appearance features. We compare our algorithm with several other algorithms on pan-tilt-zoom and baseline categories of CDnet-2014 dataset, which is a comprehensive dataset for BS evaluation [7], and Hopkins 155 dataset, which is a specialized dataset for 3D motion segmentation [8].

The contributions of the paper are twofold. First, our algorithm is based on building a flexible BG model with a comprehensive set of appearance features. It produces more robust BS as we do not rely on any global constraint in model-based algorithms or explicit camera motion models used in most existing motion-based algorithms. Second, we believe that we have provided the most comprehensive evaluation of moving-camera BS algorithms to date in terms of the size of the dataset and the number of state-of-art algorithms compared. We hope that this forms the benchmark for future research in this area.

The rest of the paper is organized as follows: we first discuss related work in Section 2. We then present the proposed method in Section 3, followed by experiments and comparison in Sections 4 and 5. Finally, we conclude the paper in Section 6.

\section{Related Work}

In this section, we review1) model-based, 2) motion-based, and 3) hybrid motion and model based BS algorithms.

\subsection{Model-based Algorithms}

Most BS algorithms construct statistical models of the background scene. The statistics can range from simple mean to complex multi-modal distributions. In general, we can classify different statistical models into two categories: pixel-based and region based.

Pixel-based algorithms form a statistical model for each pixel in an image by considering its color only. The most popular algorithms in this category are Gaussian Mixture Model (GMM) [9, 10, 11] and Kernel Density Estimates (KDE) [12, 13]. GMM models each pixel distribution using a mixture of Gaussians. In [14] authors introduce shareable GMM models. Each pixel dynamically searches for the best matched model in its neighborhood, which is then used for classification. Many variants of GMM based methods have been proposed and they are summarized in [1].

KDE accumulates pixel's recent history and estimates a nonparametric probability distribution for each pixel. This approach overcomes the problem of determining the appropriate number of components used in GMM. Sample consensus is another non-parametric method that relies on recently observed pixels to determine if the incoming pixel is a FG or BG. PAWCS is an example of sample consensus methods that introduces word based model capable of capturing and retaining color and Local Binary Similarity Pattern (LBSP) features over long periods of time. A novel pixel-level feedback loop mechanism is an integral part of the system, which allows to continuously update and maintain the pixels model [15].

Pixel based approaches suffer from the loss of inter-pixel spatial dependencies. In addition, these methods need to constantly update the distribution parameters or model itself. However, it is difficult to determine an appropriate update rate to differentiate true foreground from drastic background changes such as those caused by sudden illumination changes or moving camera.

Region Based algorithms are based on the assumption that the pixel undergoes the same change as its neighbor. Hence, they take into account inter-pixel spatial dependencies. In [16], the authors incorporate spatial information by using statistical circular shift moments (SCSM) in image regions. In [17], the authors present a block based approach that compares a block in current frame to its reconstruction from PCA coefficients, and labels it as BG if the reconstruction is close. In [18], the authors consider a $5 \times 5$ grid to compute local binary pattern features and combine them with color. Another approach is presented in [19] in which the authors not only consider the history of intensity values of pixel itself but also its neighbors. Although region based methods take into account the inter-pixel spatial dependencies, they typically assume static camera and fail to handle videos from moving camera as there is no explicit mechanism to account for the movement of the BG regions.

Apart from pixel and region based methods, hybrid approaches such as [20] also exist. The authors construct multiple average BG models of the scene based on similarity at frame level and classification at pixel level. Multiple models allow to handle camera jitter and dynamic background scenarios more robustly. Despite multiple model approach it is limited in terms of the number of scenes it can capture such as in case of a continuously panning camera. Additionaly, increasing number of models entail increased computational cost.

Our approach relies on the use of machine learning methods such as SVM for classifying features into BG or FG. There are a number of works that have taken a similar approach [21, 22, 23, 24, 25]. In [22] a one-class SVM is trained for each pixel over a number of frames. [21] proposes a single class SVM for handling dynamic background by extracting features from the neighborhood around each pixel. [24] divides an image into a predefined number of equally sized blocks and trains one class SVM for each block. [25] is another blockbased method that builds and maintains model for each block. These methods train models for each pixel or block thereby imposing spatial constraint and therefore prone to failure in case of a moving camera. In [23], the authors tackle the moving camera problem by introducing two one-class SVMs to separately model FG and BG color distributions for each pixel. Unlike our frame-based approach, their classifier is trained on local neighborhood, making it both computationally expensive and brittle for large camera movements. Additionally, the need for manual labeling limits its application for real world scenarios. 


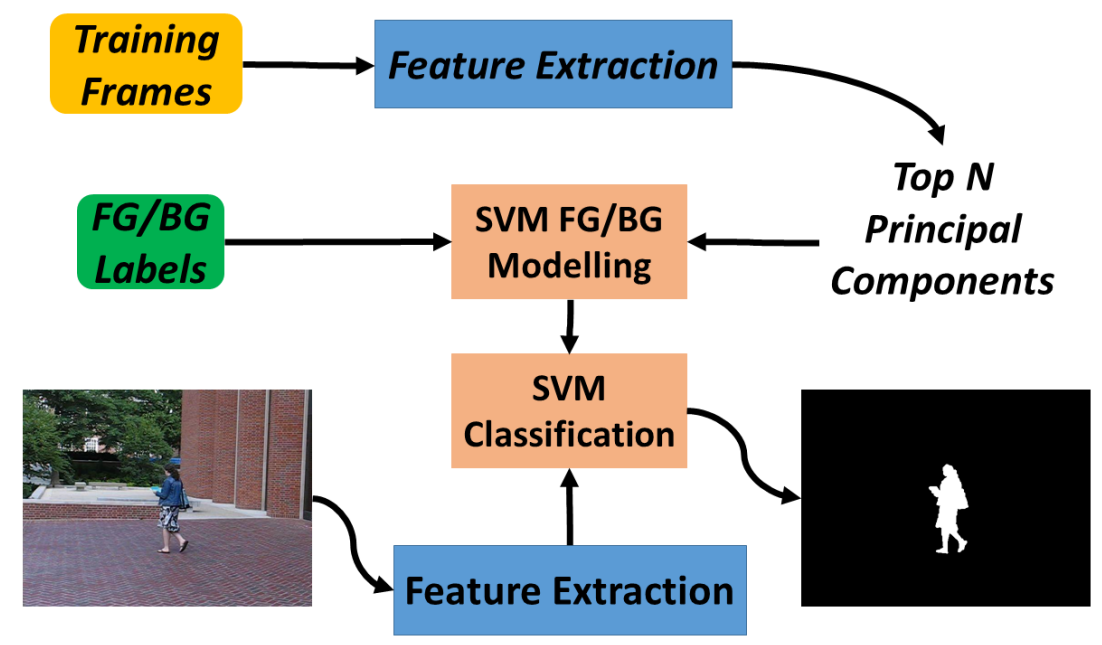

Figure 1: BoFs-SVM system

\subsection{Motion-based Algorithms}

Motion-based algorithms exploit the different motion patterns of FG and the BG. They are more commonly referred to as motion segmentation. In motion segmentation, the FG objects are continuously present in the scene, and the BG may also move due to camera motion. Motion-based algorithms can be divided into three categories: ego-motion compensation, layerbased and point trajectory based.

The ego-motion compensation based algorithms estimate the background through ego-motion compensation [26, 27, 28, 29, 30, 31, 32, 33]. These methods estimate camera motion and then apply conventional BS algorithms to detect FG. Earlier work models the BG as a single plane and assume no movement in the camera center [26, 27, 28, 29, 30]. More recent work estimate homography between image frames, followed by registration process to compensate motion [31, 32, 33]. Residual pixels can be further registered using parallax estimation [31].

Layer-based methods [34, 35, 36, 37, 38, 39, 40, 41, 42] use dense or sparse optical flows and then cluster them as piecewise planar scenes. Clustering is based on some measure of motion consistency. Although good results have been achieved, these methods are restricted by the need of an initialization step to establish prior information such as contours, number of objects, etc.

Lastly, methods such as [8, 43, 44] segment images based on point trajectory analysis. First, sparse feature points are detected and tracked in a sequence followed by clustering via spectral [45] or subspace [46] clustering. Although these methods are robust against large camera motion, they only produce a segmentation of sparse points, which need to be post-processed for dense segmentation [45]. Hence, the results of these algorithms rely heavily on point tracking throughout the video sequence and post-processing for dense segmentation.

\subsection{Motion and Model based Algorithms}

Motion and model based algorithms such as [5] and [6] exploit motion information to maintain and continuously update
FG and BG appearence models. [5] constructs FG and BG appearence models and propogates them sequentially through bayesian filtering. The noisy optical flow based motion information is robustly estimated through Nonparametric Belief Propagation (NBP). Motion is used to update and integrate previous appearence models and current frame sequentialy by bayesian filtering. [6] learns pixel based models for both FG and $\mathrm{BG}$ regions. It exploits long term trajectories for motion estimation and a Bayesian filtering framework to build appearence models. In general, these methods require special initialization procedures and are computationally expensive.

\section{Proposed Approach}

The proposed approach estimates a global FG/BG appearance model to handle videos captured from moving cameras. Our algorithm assumes the following:

1. The dominant motion from the scene is due to the camera's movement.

2. A small number of training frames, which can be externally provided or obtained through motion segmentation, are needed.

As a consequence of assumption 2, the proposed algorithm works best when the moving BG scene is predictable, such as those from a pan-tilt-zoom camera. Other moving cameras such as ego-vision cameras would require frequent retraining and adaptation, which is beyond the scope of this work. The proposed algorithm has three main components: Feature extraction, Model formation and Classification. Each component and its functional role are detailed in following subsections. Figure 1 provides the system overview.

\subsection{Feature Extraction}

This component is responsible to extract features and perform PCA to select the most significant principal components. For each pixel, a number of image features are extracted from 
the neighborhood of size $n \times n$ pixels. We use four types of appearance-based features: color, intensity, gradient and texture.

A color feature vector of size $n \times n \times 3$ is formed by concatenating three color channels: R, G and B. Likewise, intensity is the grayscale values of $n \times n$ neighborhood resulting in a feature vector of size $n \times n$. Texture features are based on Local Binary Patterns (LBP). In LBP, the center pixel is compared with its eight neighbors and a label of ' 1 ' is assigned if the center pixel is greater than the neighboring pixel and ' 0 ' otherwise. This results in an 8-bit binary pattern. Histogram of these patterns is then calculated for all the pixels in the neighborhood, which is then used as a texture descriptor. Multiple improvements and variations such as reduction in feature vector length, invariance to change of scale and rotation as well as robustness against noise and illumination changes have been reported in literature $[47,48]$. We use the scale and rotation invariant uniform LBPs introduced in [49] and its implementation in [50]. This particular choice allows us to meet real time requirements while incorporating texture information into our algorithm. Based on a recent comparison of different LBP schemes in [48], the chosen LBP implementation has significant computational advantage over its counterparts.

A gradient feature vector is formed by first calculating spatial gradients in both $\mathrm{x}$ and $\mathrm{y}$ directions using the Sobel operator. The magnitudes and directions of the gradient vectors are then used to form feature vectors of size $n \times n \times 2$ at each pixel location.

The choice of window parameter $n$ is critical since a smaller neighborhood can generalize well to different background scenes but can easily lead to frivolous matches. A larger neighborhood can capture unique features for specific background regions but become useless when those regions are no longer in the field of view. We select a $7 \times 7$ neighborhood that represents an empirically-optimal compromise for the sequences we have tested.

To find the optimal set of features, the extracted features undergo PCA and we retain the top components. The number of components is based on the percentage of variance explained by the selected subset of principal components, which must exceed a pre-defined var_threshold parameter. This rich ensemble of selected features are then combined together to form a Bag-of-Features (BoFs). Our use of BoFs capture only the local contexts which, unlike global spatial contexts, are invariant to small to medium camera and object movement. Nonetheless, local context would fail in the case of drastic scene changes such as going from outdoor to indoor.

\subsection{Model Formation}

In this step, feature vectors encoded into Bag-ofFeatures(BOFS) along with their FG/BG labels are used to train a single SVM classifier with 5-fold cross validation. The choice of SVM is deliberate as the high dimensional feature vectors from different image attributes and large patch size can easily lead to over-fitting problems. SVM classifiers provide automatic safeguard against over-fitting.
The training data comprises of feature vectors of input images and corresponding FG/BG label for each pixel. To automatically generate $\mathrm{FG} / \mathrm{BG}$ labels, a simple motion based method proposed in [51] is employed. It uses optical flow to extract motion vectors, which are then clustered into FG and BG using Gaussian Mixture Model, with the larger one labeled as BG. Apart from the aforementioned unsupervised mode, the proposed method can also take advantage of supervised mode i.e. manually label images yielding more accurate results.

These feature vectors and labels are then used to train single SVM. Using LIBSVM [52], the problem is formulated as a two-class soft-margin Support Vector Classification with regularization parameter $C$. The kernel is set to be the radial basis function $(\mathrm{RBF})$ defined as follows:

$$
K\left(x_{i}, x_{j}\right)=e^{-\gamma\left\|x_{i}-x_{j}\right\|^{2}}
$$

The setting of parameters $C$ and shape parameter $\gamma$ is based on the combination that yields the best overall performance with a 5-fold cross-validation over training data. During each iteration of the cross validation process, $10 \%$ of data is randomly retained for validation purpose, whereas remaining data is used to train the SVM model. For further details of SVM we refer readers to [52].

\subsection{Classification}

For any given image, we extract feature vectors as described in section 3.1 and pass onto the SVM model. The model returns FG and BG probability estimates for each pixel, which are then classified into $\mathrm{FG} / \mathrm{BG}$ as follows:

$$
M(x, y)=\left\{\begin{array}{lll}
B G & \text { if } \quad P(x, y) \geq t h \\
F G & \text { if } \quad P(x, y)<t h
\end{array}\right.
$$

where $M(x, y)$ represents the binary $\mathrm{FG} / \mathrm{BG}$ decision of the pixel at location $(x, y)$ of the input image, $P(x, y)$ is the $\mathrm{BG}$ probability estimate returned by the SVM classifier, and th is an empirically-determined threshold parameter. A $7 \times 7$ median filter is applied to the binary mask to remove isolated FG pixels.

\section{Evaluation on CDnet 2014 Dataset}

In this section, we compare BoFs-SVM with state of the art algorithms on CDnet 2014 dataset pan-tilt-zoom (PTZ) and baseline (BL) categories. Our goal is to demonstrate the advantages of our algorithm on PTZ sequences over the state-of-theart from the CDnet comparison website. As static cameras are special case of moving cameras, we use the BL sequences to show that our algorithm is comparable to these algorithms as well. The dataset, parameter setting and quantitative evaluation are detailed below:

\subsection{Dataset and Evaluation Metrics}

The CDnet 2014 dataset [7] is one of the most comprehensive datasets available for evaluating BS algorithms. Table 6 details the test sequences for both PTZ and BL categories. The dataset specifies training and testing data to ensure consistency when comparing different algorithms. 


\begin{tabular}{|l|l|l|l|l|l|l|l|l|}
\hline Method & $R e$ & $S p$ & $F P R$ & $F N R$ & $P W C$ & $F M$ & $P r$ & $\operatorname{Rank}(R)$ \\
\hline \hline BoFs-SVM & 0.4551 & 0.9992 & 0.0007 & 0.5448 & 0.3762 & 0.5756 & 0.7829 & 2.71 \\
\hline EFIC [53] & 0.6880 & 0.9984 & 0.0015 & 0.3119 & 0.3298 & 0.7005 & 0.7134 & 2.14 \\
\hline PAWCS [15] & 0.7664 & 0.9811 & 0.0188 & 0.2335 & 2.0014 & 0.3004 & 0.1868 & 3.14 \\
\hline MBS [20] & 0.5168 & 0.9990 & 0.0009 & 0.4831 & 0.3661 & 0.6128 & 0.7525 & 2.57 \\
\hline SharedModel [14] & 0.6814 & 0.9674 & 0.0325 & 0.3185 & 3.4128 & 0.1829 & 0.1056 & 4.42 \\
\hline
\end{tabular}

Table 1: Results for continuousPan test sequence.

\begin{tabular}{|l|l|l|l|l|l|l|l|l|}
\hline Method & $R e$ & $S p$ & $F P R$ & $F N R$ & $P W C$ & $F M$ & $P r$ & $\operatorname{Rank}(R)$ \\
\hline \hline BoFs-SVM & 0.5633 & 0.9988 & 0.0011 & 0.4366 & 0.5275 & 0.6669 & 0.8172 & 2.57 \\
\hline EFIC [53] & 0.9070 & 0.9998 & 0.0001 & 0.0929 & 0.1039 & 0.9424 & 0.9806 & 1 \\
\hline PAWCS [15] & 0.4504 & 0.9980 & 0.0019 & 0.5495 & 0.7044 & 0.5452 & 0.6907 & 3.57 \\
\hline MBS [20] & 0.7072 & 0.9914 & 0.0085 & 0.2927 & 1.1258 & 0.5409 & 0.4379 & 3.71 \\
\hline SharedModel [14] & 0.7649 & 0.9840 & 0.0159 & 0.2350 & 1.7961 & 0.4440 & 0.3128 & 4.14 \\
\hline
\end{tabular}

Table 2: Results for intermittentPan test sequence.

\begin{tabular}{|l|l|l|l|l|l|l|l|l|}
\hline Method & $R e$ & $S p$ & $F P R$ & $F N R$ & $P W C$ & $F M$ & $P r$ & $\operatorname{Rank}(R)$ \\
\hline \hline BoFs-SVM & 0.8334 & 0.9985 & 0.0014 & 0.1665 & 0.3350 & 0.8532 & 0.8740 & 2.28 \\
\hline EFIC [53] & 0.9191 & 0.9965 & 0.0034 & 0.0808 & 0.4353 & 0.8315 & 0.7591 & 3.14 \\
\hline PAWCS [15] & 0.7414 & 0.9991 & 0.0008 & 0.2585 & 0.3887 & 0.8167 & 0.9091 & 2.71 \\
\hline MBS [20] & 0.8425 & 0.9967 & 0.0032 & 0.1574 & 0.5050 & 0.7959 & 0.7541 & 4.14 \\
\hline SharedModel [14] & 0.8770 & 0.9971 & 0.0028 & 0.1229 & 0.4288 & 0.8270 & 0.7823 & 2.71 \\
\hline
\end{tabular}

Table 3: Results for twoPositionPTZCam test sequence.

\begin{tabular}{|l|l|l|l|l|l|l|l|l|}
\hline Method & $R e$ & $S p$ & $F P R$ & $F N R$ & $P W C$ & $F M$ & $P r$ & $\operatorname{Rank}(R)$ \\
\hline \hline BoFs-SVM & 0.9362 & 0.9998 & 0.0001 & 0.0637 & 0.0298 & 0.9207 & 0.9058 & 1.28 \\
\hline EFIC [53] & 0.9601 & 0.5841 & 0.4158 & 0.0398 & 41.520 & 0.0084 & 0.0042 & 3.85 \\
\hline PAWCS [15] & 0.8322 & 0.9865 & 0.0134 & 0.1677 & 1.3700 & 0.1835 & 0.1031 & 3.28 \\
\hline MBS [20] & 0.3226 & 0.9978 & 0.0021 & 0.6773 & 0.3427 & 0.2583 & 0.2153 & 2.85 \\
\hline SharedModel [14] & 0.8644 & 0.9679 & 0.0320 & 0.1355 & 3.2286 & 0.0901 & 0.0475 & 3.71 \\
\hline
\end{tabular}

Table 4: Results for zoomInZoomOut test sequence.

\begin{tabular}{|l|l|l|l|l|l|l|l|l|}
\hline Method & $R e$ & $S p$ & $F P R$ & $F N R$ & $P W C$ & $F M$ & $P r$ & $\operatorname{Rank}(R)$ \\
\hline \hline BoFs-SVM & 0.6970 & 0.9991 & 0.0008 & 0.3029 & 0.3171 & 0.7541 & 0.8450 & 1.85 \\
\hline EFIC [53] & 0.8686 & 0.8947 & 0.1052 & 0.1313 & 10.597 & 0.6207 & 0.6143 & 3 \\
\hline PAWCS [15] & 0.6976 & 0.9912 & 0.0087 & 0.3023 & 1.1161 & 0.4615 & 0.4724 & 3.28 \\
\hline MBS [20] & 0.5973 & 0.9962 & 0.0037 & 0.4026 & 0.5849 & 0.5519 & 0.5400 & 3.14 \\
\hline SharedModel [14] & 0.7969 & 0.9791 & 0.0208 & 0.2030 & 2.2166 & 0.3860 & 0.3121 & 3.71 \\
\hline \multicolumn{8}{|c|}{ Table 5: Overall comparison on PTZ Category. }
\end{tabular}

In addition, CDnet recommends seven evaluation metrics. Let $\mathrm{TP}=$ True Positive, $\mathrm{FP}=$ False Positive, $\mathrm{TN}=$ True Negative and $\mathrm{TP}=$ True Positive. The metrics are defines as:

1. Recall: $R e=\frac{T P}{T P+F N}$

2. Specificity: $S p=\frac{T N}{T N+F P}$

3. False Positive Rate: $F P R=\frac{F P}{F P+T N}$

4. False Negative Rate: $F N R=\frac{F N}{F P+T N}$

5. Percentage of Wrong Classifications: $P W C=100$. $\frac{F N+F P}{F N+F P+T N+T P}$

6. Precision: $P r=\frac{T P}{T P+F P}$

7. F-Measure: $F M=\frac{2 P r \cdot R e}{P r+R e}$

An additional metric called average rank $R$ is also defined to aggregate all seven metrics together, which is simply the average of each metric from all 4 test sequences.

\subsection{Parameter Selection}

In order to systematically find the optimal set of parameters, exhaustive search is conducted during the offline training phase to identify the optimal $C$, th, var_threshold and $\gamma$. The set of

\begin{tabular}{|l|l|l|l|}
\hline Test Sequence & Resolution & $\begin{array}{l}\text { Training } \\
\text { (Frame \#s) }\end{array}$ & $\begin{array}{l}\text { Testing } \\
\text { (Frame \#s) }\end{array}$ \\
\hline \hline PTZ - continuousPan & $704 \times 480$ & $1-599$ & $600-1700$ \\
\hline PTZ - intermittentPan & $560 \times 368$ & $1-1199$ & $1200-3500$ \\
\hline PTZ - twoPositionPTZ & $570 \times 340$ & $1-799$ & $800-2300$ \\
\hline PTZ - zoomInZoomOut & $320 \times 240$ & $1-499$ & $500-1130$ \\
\hline BL - highway & $320 \times 240$ & $1-469$ & $470-1700$ \\
\hline BL - office & $360 \times 240$ & $1-569$ & $570-2050$ \\
\hline BL - pedestrians & $360 \times 240$ & $1-299$ & $300-1099$ \\
\hline BL - PETS2006 & $720 \times 576$ & $1-299$ & $300-1200$ \\
\hline
\end{tabular}

Table 6: CDnet 2014 PTZ test sequence details.

parameters that yields the best result in terms of F-Measure over the validation data is chosen.

This process has resulted in a single set of parameters to be used for all PTZ sequences in CDnet2014 dataset: $C=1$, th $=$ 0.97 , var_threshold $=90 \%$ and $\gamma=0.25$.

\subsection{Quantitative Evaluation}

For quantitative evaluation we consider the top 4 methods reported on the CDnet website in PTZ category: EFIC [53], PAWCS [15], MBS [20] and SharedModel [14]. Tables 1 to 4 


\begin{tabular}{|l|l|l|l|l|l|l|l|l|}
\hline Method & $R e$ & $S p$ & $F P R$ & $F N R$ & $P W C$ & $F M$ & $P r$ & $\operatorname{Rank}(R)$ \\
\hline \hline BoFs-SVM & 0.9115 & 0.9978 & 0.0020 & 0.0884 & 0.4606 & 0.9210 & 0.9308 & 4.14 \\
\hline EFIC [53] & 0.9455 & 0.9970 & 0.0030 & 0.0545 & 0.5201 & 0.9309 & 0.9170 & 3.85 \\
\hline PAWCS [15] & 0.9408 & 0.9980 & 0.0020 & 0.0592 & 0.4491 & 0.9397 & 0.9394 & 2.71 \\
\hline MBS [20] & 0.9158 & 0.9979 & 0.0021 & 0.0842 & 0.4361 & 0.9287 & 0.9431 & 3.28 \\
\hline SharedModel [14] & 0.9545 & 0.9982 & 0.0018 & 0.0455 & 0.3344 & 0.9522 & 0.9502 & 1 \\
\hline \multicolumn{7}{|c|}{ Table 7: Ovell comparison on Baseline Category. }
\end{tabular}

Table 7: Overall comparison on Baseline Category.

contain the results of BoFs-SVM and the top 4 algorithms on four test sequences, whereas the overall results are presented in Table 5. It is important to note that the results reported in this paper are official results computed by the CDnet administrator based on our submission of the binary masks. The ground-truth used in the evaluation are withheld by the administrator and unavailable to us.

Our algorithm ranks first for both ZoomInZoomOut and twoPositionPTZCam test sequences, second for intermittentPan test sequence, and third for continuousPan test sequence. The results in intermittentPan and continuousPan test sequences is affected by the presence of parked cars in the scene. The lack of spatial constraint and the similarity in features of the parked cars and moving FG cars decreases the segmentation accuracy. The overall comparison in Table 5 clearly indicates the superiority of our proposed algorithm: it ranks first in five out of seven metrics and yields comparable results in the Re and FNR metrics. In terms of the F-Measure, we have achieved $13.34 \%$ improvement over previous best score of $62.07 \%$. Most of the aforementioned methods fail because of underlying static camera assumption and spatial constraint, whereas our algorithm does not impose any spatial constraint and therefore produces significantly more accurate results. Figures 2 and 3 show qualitative results of BoFs-SVM for PTZ test sequences. Note the challenging nature and large camera motion in these test sequences. Complete video results are provided as part of supplementary materials.

Table 7 details the overall results on baseline category of CDnet dataset. Although our algorithm does not outperform other methods. It produces comparable results across all seven metrics. Specifically, our algorithm produces a FM of 0.92 in comparison to top perfroming method with FM of 0.95 .

In order to highlight the importance of appropriate feature selection, we also tested our algorithm with color, LBP and gradient features individually. The results are detailed in Table 8.

\begin{tabular}{|l|l|l|l|}
\hline Method & Pr & Re & FM \\
\hline \hline BoFs-SVM-Gradient & 0.215 & 0.356 & 0.268 \\
\hline BoFs-SVM-LBP & 0.642 & 0.421 & 0.507 \\
\hline BoFs-SVM-Color & $\mathbf{0 . 8 3 9}$ & $\mathbf{0 . 6 2 3}$ & $\mathbf{0 . 6 9 0}$ \\
\hline
\end{tabular}

Table 8: Overall Results with different features on CDnet 2014 PTZ category.

Clearly, color features offer significantly more robust solution than LBP and gradient feature vectors. The results for texture (LBP) and color features are affected by two test sequences in PTZ category: continuousPan and intermittentPan. Due to lack of spatial constraint, the proposed method is unable to distinguish moving cars in the foreground and parked cars in the background. The same texture of parked and moving cars results in poor performance when LBP features are chosen, however color features produce more accurate results since the color of moving and parked cars are not necessarily the same. This stresses the need to choose correct type of features depending on the type of the scene.

Lastly, we compare the processing time of our algorithm with other methods in table 9. The processing time for other methods are reported from official CDnet dataset website [7]. Our MATLAB implementation of the proposed method is able to acheive 15 frames per second (fps).

\begin{tabular}{|l|l|l|l|}
\hline Method & Implementation & Resolution & fps \\
\hline \hline BoFs-SVM & Matlab & $320 \times 240$ & 15 \\
\hline EFIC [53] & C++ & $320 \times 240$ & 16 \\
\hline PAWCS [15] & C++ & $320 \times 240$ & 27 \\
\hline MBS [20] & Matlab & $320 \times 240$ & 9 \\
\hline SharedModel [14] & C++ & $320 \times 240$ & 35 \\
\hline
\end{tabular}

Table 9: Processing time comparison.

\section{Evaluation on Hopkins155 Dataset}

In this section, we compare BoFs-SVM with four state of the art algorithms on Hopkins 155 dataset. The dataset, parameter setting and quantitative evaluation are detailed below.

\subsection{Dataset and Evaluation Metrics}

The reason to include this particular dataset is that these test sequences are taken from hand-held cameras and often used in motion segmentation literature. Although, Hopkins 155 dataset comprises of 26 video sequences, most literature focuses on three test sequences: cars1, people1 and people2, since majority of test sequences have large number of frames with zero motion and despite multiple moving objects, only one or few are labeled for evaluation.

Hopkins 155 dataset has no FG-free or static frames for training purposes. Therefore similar to other algorithms, we simply apply optical flow on the first two frames of the sequence to obtain initial labels and train the SVM classifier. Additionally, we report the supervised mode of our algorithm for which we manually label the first frame of a sequence. Precision(Pr), Recall(Re) and F-Measure(FM) are used for evaluation purposes.

\subsection{Parameter Selection}

Exhaustive search is conducted during the offline training phase to identify the optimal $C, t h$, var_threshold and $\gamma$. The 


\begin{tabular}{|l|c|c|c|c|c|c|c|c|c|c|c|c|}
\hline & \multicolumn{3}{|c|}{ Cars1 } & \multicolumn{3}{c|}{ People1 } & \multicolumn{3}{c|}{ People2 } & \multicolumn{1}{c|}{ Overall } \\
\hline Method & $P r$ & $R e$ & $F M$ & $P r$ & $R e$ & $F M$ & $P r$ & $R e$ & $F M$ & $P r$ & $R e$ & $F M$ \\
\hline BoFs-SVM & 0.81 & 0.92 & 0.86 & 0.87 & 0.88 & 0.87 & 0.83 & 0.93 & 0.88 & 0.83 & 0.91 & 0.87 \\
\hline Brox and malik [44] & - & - & - & 0.89 & 0.77 & 0.83 & 0.92 & 0.89 & 0.90 & - & - & - \\
\hline Kwak et al. [5] & 0.92 & 0.84 & 0.88 & 0.95 & 0.93 & 0.94 & 0.85 & 0.89 & 0.87 & 0.90 & 0.88 & 0.89 \\
\hline Sheikh et al. [43] & 0.63 & 0.99 & 0.77 & 0.78 & 0.63 & 0.70 & 0.73 & 0.83 & 0.77 & 0.71 & 0.81 & 0.74 \\
\hline Elqursh and Elgammal [6] & 0.85 & 0.97 & 0.90 & 0.97 & 0.88 & 0.92 & 0.87 & 0.88 & 0.87 & 0.89 & 0.91 & 0.89 \\
\hline
\end{tabular}

Table 10: Hopkins 155 dataset results

set of parameters that yields the best F-Measure over the validation data is chosen.

This process has resulted in a single set of parameters to be used for all of the three test sequences in Hopkins 155 dataset: $C=1$, th $=0.97$, var_threshold $=90 \%$ and $\gamma=0.25$.

\subsection{Quantitative Evaluation}

For quantitative evaluation, we consider 4 state of the art algorithms: Brox and Malik [44], Kwak et al. [5], Sheikh et al. [43], as well as Elqursh and Elgammal [6]. We tabulate individual as well as overall results in Table 10. Note that in each column of tables, red font represents the best result and blue font represents the second best.

For cars1 test sequence, our algorithm produces a FM of 0.86 , which is comparable to FM of 0.88 produced by the second best method Kwak et al. [5]. The slightly poorer result is due to the limitation of the training data, which are obtained in our experiments based on motion segmentation of first two frames. For cars1, part of the FG has the same motion as the $\mathrm{BG}$ and this contributes to the slight loss in performance.

In people 1 test sequences, our algorithm has second best recall, whereas for people 2 test sequence, it acheives highest recall and second best F-Measure of 0.88. It is important to mention that our current system uses only one set of parameters to train SVM model for consistency. Table 10 shows that the proposed method achieves the highest overall recall and second best F-Measure of 0.87, which is comparabale to top F-Measure of 0.89. Lastly, Figure 4 depicts results of BoFs-SVM for the three test sequences of Hopkins155 dataset. Complete video results are provided as a part of supplementary material.

We also tested our algorithm with color, LBP and gradient features individually. Table 11 details the overall performance on Hopkins 155 dataset. Like CDnet 2014, color features offer the most robust segmentation. LBP and gradient feature vectors yield better results on Hopkins 155 in comparison to their performance on CDnet 2014 dataset since Hopkins 155 does not involve large camera movements as that of CDnet 2014 and FG objects similar to BG do not appear in the scene.

\begin{tabular}{|l|l|l|l|}
\hline Method & Pr & Re & FM \\
\hline \hline BoFs-SVM-Gradient & 0.551 & 0.426 & 0.480 \\
\hline BoFs-SVM-LBP & 0.672 & 0.598 & 0.632 \\
\hline BoFs-SVM-Color & $\mathbf{0 . 9 0 4}$ & $\mathbf{0 . 8 8 5}$ & $\mathbf{0 . 8 9 4}$ \\
\hline
\end{tabular}

Table 11: Overall Results with different features on Hopkins 155 dataset.

\section{Conclusion and Future Work}

We have presented a novel background subtraction algorithm for PTZ cameras. The proposed method extracts multiple features at each pixel, which then undergoes PCA. A subset of top principal components are retained based on the amount of variance and then encoded into a Bag-of-Features to represent background without any spatial constraint. These features are then used to train a single SVM classifier for labeling pixels. The same set of features for all test sequences indicates the strength and applicability of proposed method for real world scenarios. Evaluation of the proposed method on CDnet 2014 and Hopkins 155 dataset have demonstrated superiority in comparison to both model-based, motion-based and hybrid motion and model based algorithms.

The current version of proposed algorithm lacks model update. Incorporation of a model update mechanism and $\mathrm{C}++\mathrm{im}-$ plementation of proposed method are a part of our future work.

\section{Acknowledgment}

Part of this material is based upon work supported by the National Science Foundation under Grant No. 1237134. Any opinions, findings, and conclusions or recommendations expressed in this material are those of the author(s) and do not necessarily reflect the views of the National Science Foundation.

\section{References}

[1] T. Bouwmans, Recent advanced statistical background modeling for foreground detection-a systematic survey, Recent Patents on Computer Science 4 (3) (2011) 147-176.

[2] T. B. Moeslund, A. Hilton, V. Krüger, A survey of advances in visionbased human motion capture and analysis, Computer vision and image understanding 104 (2) (2006) 90-126.

[3] A. Yilmaz, O. Javed, M. Shah, Object tracking: A survey, Acm computing surveys (CSUR) 38 (4) (2006) 13.

[4] V. Nair, J. J. Clark, An unsupervised, online learning framework for moving object detection, in: Computer Vision and Pattern Recognition, 2004. CVPR 2004. Proceedings of the 2004 IEEE Computer Society Conference on, Vol. 2, IEEE, 2004, pp. II-317.

[5] S. Kwak, T. Lim, W. Nam, B. Han, J. H. Han, Generalized background subtraction based on hybrid inference by belief propagation and Bayesian filtering, in: Computer Vision (ICCV), 2011 IEEE International Conference on, IEEE, 2011, pp. 2174-2181.

[6] A. Elqursh, A. Elgammal, Online moving camera background subtraction, in: Computer Vision-ECCV 2012, Springer, 2012, pp. 228-241.

[7] Y. Wang, P.-M. Jodoin, F. Porikli, J. Konrad, Y. Benezeth, P. Ishwar, Cdnet 2014: An expanded change detection benchmark dataset, in: Computer Vision and Pattern Recognition Workshops (CVPRW), 2014 IEEE Conference on, IEEE, 2014, pp. 393-400. 

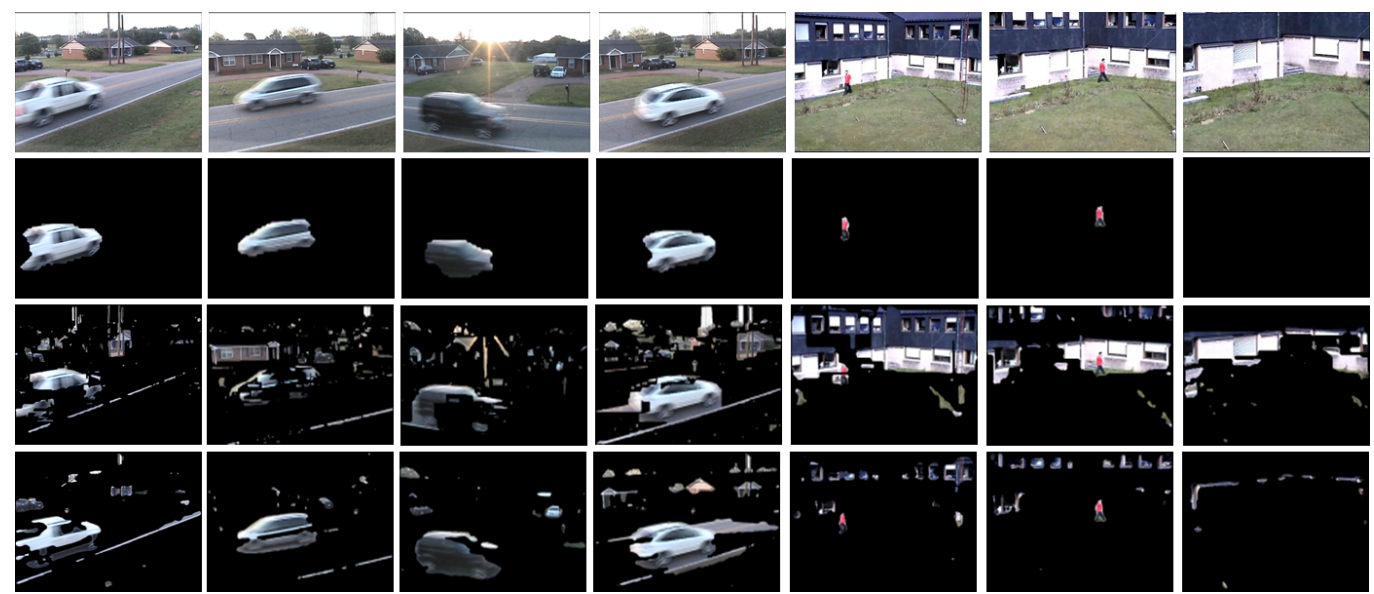

Figure 2: Input Image (row 1), BoFs-SVM output (row 2), EFIC output(row 3), and PAWCS output(row 4). CDnet 2014 dataset: continuousPan test sequence(columns 1-4) and zoomInZoomOut(columns 5-7) test sequences.

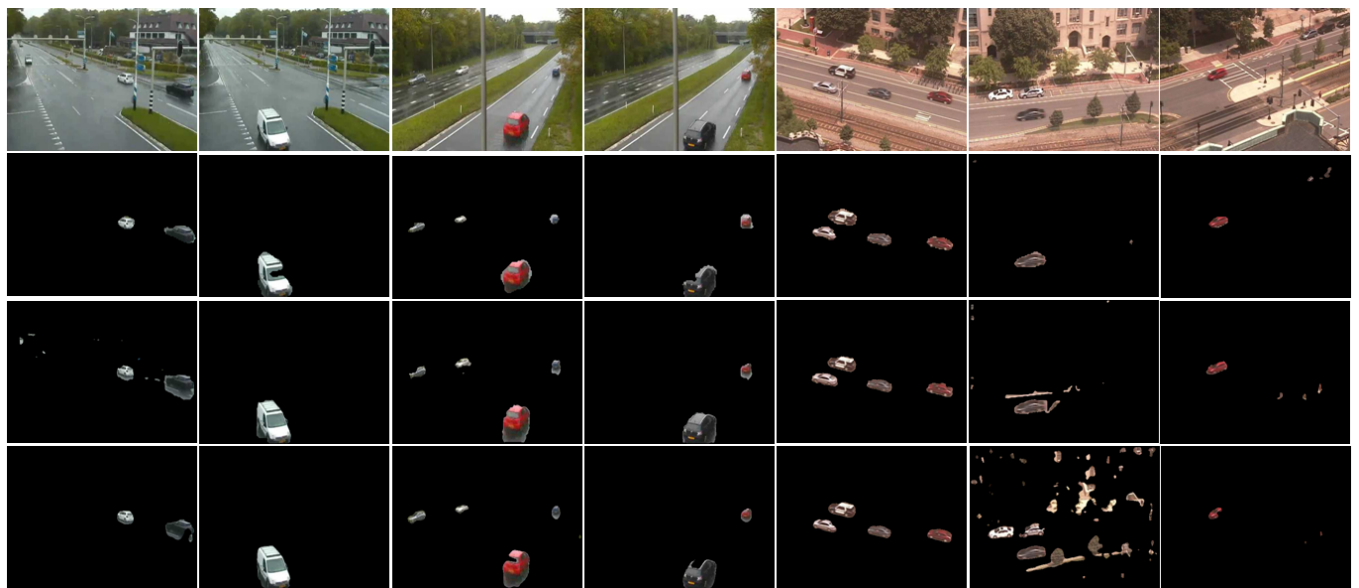

Figure 3: Input Image (row 1), BoFs-SVM output (row 2), EFIC output(row 3), and PAWCS output(row 4). CDnet 2014 dataset - twoPositionPTZCam(columns 1-4) and intermittentPan(columns 5-7) test sequences.

[8] R. Tron, R. Vidal, A benchmark for the comparison of 3-d motion segmentation algorithms, in: Computer Vision and Pattern Recognition, 2007. CVPR'07. IEEE Conference on, IEEE, 2007, pp. 1-8.

[9] C. Stauffer, W. E. L. Grimson, Adaptive background mixture models for real-time tracking, in: Computer Vision and Pattern Recognition, 1999. IEEE Computer Society Conference on., Vol. 2, IEEE, 1999.

[10] Z. Zivkovic, F. van der Heijden, Efficient adaptive density estimation per image pixel for the task of background subtraction, Pattern recognition letters 27 (7) (2006) 773-780.

[11] P. D. Z. Varcheie, M. Sills-Lavoie, G.-A. Bilodeau, A multiscale regionbased motion detection and background subtraction algorithm, Sensors 10 (2) (2010) 1041-1061.

[12] A. Elgammal, D. Harwood, L. Davis, Non-parametric model for background subtraction, in: Computer VisionECCV 2000, Springer, 2000, pp. 751-767.

[13] Y. Sheikh, M. Shah, Bayesian modeling of dynamic scenes for object detection, Pattern Analysis and Machine Intelligence, IEEE Transactions on 27 (11) (2005) 1778-1792.

[14] Y. Chen, J. Wang, H. Lu, Learning sharable models for robust background subtraction, in: Multimedia and Expo (ICME), 2015 IEEE International Conference on, IEEE, 2015, pp. 1-6.

[15] P.-L. St-Charles, G.-A. Bilodeau, R. Bergevin, A self-adjusting approach to change detection based on background word consensus, in: Applications of Computer Vision (WACV), 2015 IEEE Winter Conference on, IEEE, 2015, pp. 990-997.

[16] S.-C. Liu, C.-W. Fu, S. Chang, Statistical change detection with moments under time-varying illumination, Image Processing, IEEE Transactions on 7 (9) (1998) 1258-1268.

[17] M. Seki, T. Wada, H. Fujiwara, K. Sumi, Background subtraction based on cooccurrence of image variations, in: Computer Vision and Pattern Recognition, 2003. Proceedings. 2003 IEEE Computer Society Conference on, Vol. 2, IEEE, 2003, pp. II-65.

[18] P.-L. St-Charles, G.-A. Bilodeau, R. Bergevin, Subsense: A universal change detection method with local adaptive sensitivity, Image Processing, IEEE Transactions on 24 (1) (2014) 359-373.

[19] O. Barnich, M. Van Droogenbroeck, Vibe: A universal background subtraction algorithm for video sequences, Image Processing, IEEE Transactions on 20 (6) (2011) 1709-1724.

[20] H. Sajid, S.-C. S. Cheung, Background subtraction for static \& moving camera, in: Image Processing (ICIP), 2015 IEEE International Conference on, IEEE, 2015, pp. 4530-4534.

[21] I. Junejo, A. Bhutta, H. Foroosh, Single Class Support Vector Machine (SVM) for scene modeling, Journal of Signal, Image and Video Processing, Springer-Verlag.

[22] L. Cheng, S. Wang, D. Schuurmans, T. Caelli, S. Vishwanathan, An online discriminative approach to background subtraction, in: Video and Signal Based Surveillance, 2006. AVSS'06. IEEE International Conference on, IEEE, 2006, pp. 2-2.

[23] M. Gong, L. Cheng, Foreground segmentation of live videos using locally competing 1SVMs, in: Computer Vision and Pattern Recognition (CVPR), 2011 IEEE Conference on, IEEE, 2011, pp. 2105-2112.

[24] A. Glazer, M. Lindenbaum, S. Markovitch, One-class background model, in: Computer Vision-ACCV 2012 Workshops, Springer, 2012, pp. 301307. 

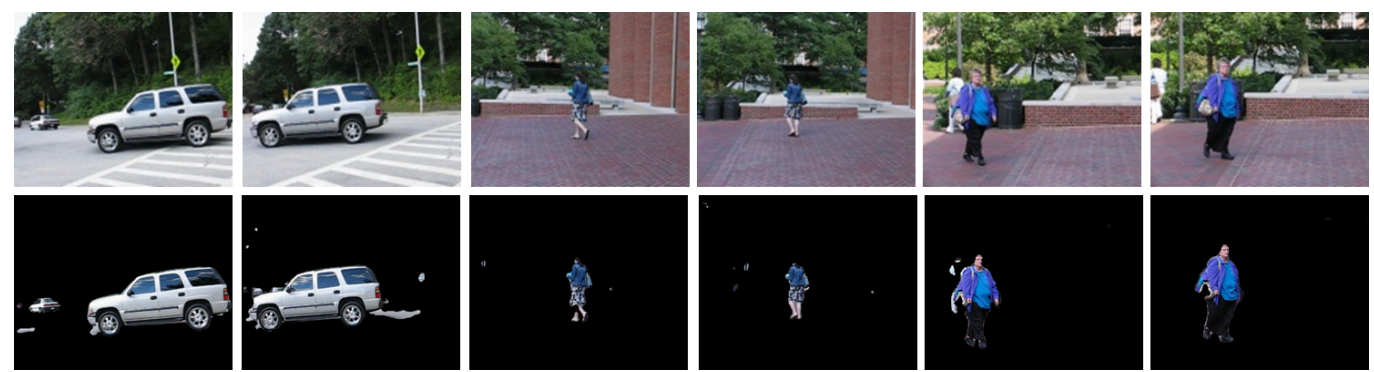

Figure 4: Input Image (row 1) and BoFs-SVM output(row 2). Hopkins15

dataset: Cars1(column 1-2), people1(column 3-4) and people2(column 5-6).

[25] H.-H. Lin, T.-L. Liu, J.-H. Chuang, Learning a scene background model via classification, Signal Processing, IEEE Transactions on 57 (5) (2009) $1641-1654$

[26] E. Hayman, J.-O. Eklundh, Statistical background subtraction for a mobile observer, in: Computer Vision, 2003. Proceedings. Ninth IEEE International Conference on, IEEE, 2003, pp. 67-74.

[27] A. Mittal, D. Huttenlocher, Scene modeling for wide area surveillance and image synthesis, in: Computer Vision and Pattern Recognition, 2000. Proceedings. IEEE Conference on, Vol. 2, IEEE, 2000, pp. 160-167.

[28] M. Irani, B. Rousso, S. Peleg, Computing occluding and transparent motions, International Journal of Computer Vision 12 (1) (1994) 5-16.

[29] Y. Ren, C.-S. Chua, Y.-K. Ho, Statistical background modeling for nonstationary camera, Pattern Recognition Letters 24 (1) (2003) 183-196.

[30] S. Rowe, A. Blake, Statistical mosaics for tracking, Image and Vision Computing 14 (8) (1996) 549-564.

[31] C. Yuan, G. Medioni, J. Kang, I. Cohen, Detecting motion regions in the presence of a strong parallax from a moving camera by multiview geometric constraints, Pattern Analysis and Machine Intelligence, IEEE Transactions on 29 (9) (2007) 1627-1641.

[32] M. Irani, P. Anandan, A unified approach to moving object detection in 2d and $3 \mathrm{~d}$ scenes, Pattern Analysis and Machine Intelligence, IEEE Transactions on 20 (6) (1998) 577-589.

[33] H. S. Sawhney, Y. Guo, J. Asmuth, R. Kumar, Independent motion detection in 3d scenes, in: Computer Vision, 1999. The Proceedings of the Seventh IEEE International Conference on, Vol. 1, IEEE, 1999, pp. 612619.

[34] J. Y. Wang, E. H. Adelson, Representing moving images with layers, Image Processing, IEEE Transactions on 3 (5) (1994) 625-638.

[35] D. Cremers, S. Soatto, Motion competition: A variational approach to piecewise parametric motion segmentation, International Journal of Computer Vision 62 (3) (2005) 249-265.

[36] M. J. Black, P. Anandan, The robust estimation of multiple motions: Parametric and piecewise-smooth flow fields, Computer vision and image understanding 63 (1) (1996) 75-104.

[37] T. Amiaz, N. Kiryati, Piecewise-smooth dense optical flow via level sets, International Journal of Computer Vision 68 (2) (2006) 111-124.

[38] T. Brox, A. Bruhn, J. Weickert, Variational motion segmentation with level sets, in: ECCV 2006, Springer, 2006, pp. 471-483.

[39] M. P. Kumar, P. H. Torr, A. Zisserman, Learning layered motion segmentations of video, International Journal of Computer Vision 76 (3) (2008) 301-319.

[40] H. Tao, H. S. Sawhney, R. Kumar, Object tracking with bayesian estimation of dynamic layer representations, Pattern Analysis and Machine Intelligence, IEEE Transactions on 24 (1) (2002) 75-89.

[41] Y. Weiss, Smoothness in layers: Motion segmentation using nonparametric mixture estimation, in: Computer Vision and Pattern Recognition, 1997. Proceedings., 1997 IEEE Computer Society Conference on, IEEE, 1997, pp. 520-526.

[42] J. Xiao, M. Shah, Accurate motion layer segmentation and matting, in: Computer Vision and Pattern Recognition, 2005. CVPR 2005. IEEE Computer Society Conference on, Vol. 2, IEEE, 2005, pp. 698-703.

[43] Y. Sheikh, O. Javed, T. Kanade, Background subtraction for freely moving cameras, in: Computer Vision, 2009 IEEE 12th International Conference on, IEEE, 2009, pp. 1219-1225.

[44] T. Brox, J. Malik, Object segmentation by long term analysis of point trajectories, in: ECCV 2010, Springer, 2010, pp. 282-295.
[45] P. Ochs, T. Brox, Object segmentation in video: a hierarchical variational approach for turning point trajectories into dense regions, in: Computer Vision (ICCV), 2011 IEEE International Conference on, IEEE, 2011, pp. 1583-1590.

[46] R. Vidal, A tutorial on subspace clustering, IEEE Signal Processing Magazine 28 (2) (2010) 52-68.

[47] D. Huang, C. Shan, M. Ardabilian, Y. Wang, L. Chen, Local binary patterns and its application to facial image analysis: a survey, Systems, Man, and Cybernetics, Part C: Applications and Reviews, IEEE Transactions on 41 (6) (2011) 765-781.

[48] C. Silva, T. Bouwmans, C. Frélicot, An extended center-symmetric local binary pattern for background modeling and subtraction in videos, in: International Joint Conference on Computer Vision, Imaging and Computer Graphics Theory and Applications, VISAPP 2015, 2015.

[49] T. Ojala, M. Pietikainen, T. Maenpaa, Multiresolution gray-scale and rotation invariant texture classification with local binary patterns, Pattern Analysis and Machine Intelligence, IEEE Transactions on 24 (7) (2002) 971-987.

[50] A. Vedaldi, B. Fulkerson, Vlfeat: An open and portable library of computer vision algorithms, in: Proceedings of the international conference on Multimedia, ACM, 2010, pp. 1469-1472.

[51] H. Sajid, S.-c. S. Cheung, Vsig: Hand-gestured signature recognition and authentication with wearable camera, in: Information Forensics and Security (WIFS), 2015 IEEE International Workshop on, IEEE, 2015, pp. $1-6$.

[52] C.-C. Chang, C.-J. Lin, Libsvm: a library for support vector machines, ACM Transactions on Intelligent Systems and Technology (TIST) 2 (3) (2011) 27.

[53] G. Allebosch, D. V. Hamme, F. Deboeverie, P. Veelaert, W. Philips, Edge based foreground background segmentation with interior/exterior classification, in: Proceedings of VISAPP, 2015. 